\title{
Association Between Covid-19 and Heart Failure: Evidence From a Two-sample Mendelian Randomization Study
}

\section{Huachen Wang}

Tianjin Medical University Second Hospital

\section{Zheng Guo}

Edith Cowan University

Yulu Zheng

Edith Cowan University

Bing Chen ( $\square$ cb20202023@163.com )

Tianjin Medical University Second Hospital

\section{Research}

Keywords: COVID-19, heart failure, single nucleotide polymorphisms, instrumental variable, Mendelian randomization study

Posted Date: March 11th, 2021

DOI: https://doi.org/10.21203/rs.3.rs-277791/v1

License: (c) (i) This work is licensed under a Creative Commons Attribution 4.0 International License. Read Full License 


\section{Abstract}

Background: Current research observing inconsistent associations of Corona Virus Disease 2019 (COVID19) with heart failure (HF) are prone to bias based on reverse causality and residual confounding factors. Our aim was to apply a two-sample Mendelian randomization method to investigate whether COVID-19 has a causal effect on HF.

Methods: Twenty-nine single nucleotide polymorphisms (SNPs) were proposed as candidate instrumental variables (IVs). A total of 3,523 patients with COVID-19 and 36,634 control participants were included in the genome-wide meta-analysis. We analyzed the largest genome-wide association studies (GWAS) metaanalysis of heart failure in individuals of European ancestry consisting of 47,309 patients with HF and 930,014 controls. The inverse variance weighted (IVW), the Mendelian randomization-Egger (MR-Egger) regression, the simple mode (SM), weighted median, and weighted mode were utilized for the MR analysis to test the stability and a causal effect.

Results: The IVW, MR-Egger regression, SM, weighted median and weighted mode demonstrated there was no association between the genetically predicted COVID-19 infection and HF risk (OR, $1.004 ; 95 \% \mathrm{Cl}$, 0.994-1.014; $\mathrm{P}=0.467 ; \mathrm{OR}, 1.008 ; 95 \% \mathrm{Cl}, 0.996-1.019 ; \mathrm{P}=0.218 ; \mathrm{OR}, 0.968 ; 95 \% \mathrm{Cl}, 0.924-1.015 ; \mathrm{P}=0.186$; $\mathrm{OR}, 1.001 ; 95 \% \mathrm{Cl}, 0.988-1.014 ; \mathrm{P}=0.881 ; \mathrm{OR}, 1.001 ; 95 \% \mathrm{Cl}, 0.989-1.014 ; \mathrm{P}=0.836$; respectively).

Conclusion: This two-sample Mendelian randomization analysis provided no evidence to sustain the causality of COVID-19 on HF.

\section{Introduction}

Corona virus disease 2019 (COVID-19), which is caused by the severe acute respiratory syndrome coronavirus 2 (SARS-COV2) and emerged in late 2019, quickly developed into a pandemic ${ }^{[1]}$ around the world. The clinical scope of COVID-19 infection seems to be significantly wide, including asymptomatic infection, mild or severe disease, organ failure such as heart failure (HF), and even death ${ }^{[2,3]}$. A considerable number of case studies pointed out that heart damage, such as arrhythmia, cardiac arrest, and heart failure are the end-stage events of COVID-19 patients ${ }^{[4-6]}$. The mortality rate of patients with heart injury is higher than that of patients without heart injury ${ }^{[5-7]}$. A previous study showed that the vast majority of acute heart failure cases (77.9\%) developed in COVID-19 infection patients without history of $\mathrm{HF}^{[8]}$. Another retrospective cohort study demonstrated that $52 \%$ of HF patients who infected COVID-19 were dying, $12 \%$ in those who recovered and were discharged ${ }^{[9]}$. Some researchers tried to detect the possible association between COVID-19 infection and HF ${ }^{[10,11]}$.Cytokine storm, levels of ACE2, and angiotensin II may be the cause of HF in the COVID-19 infection population ${ }^{[5,12-14]}$. Studies based on endomyocardial biopsy and macrophages showed the SARS-COV2 can reside within the heart myocardial tissue, however, it does not prove SARS-COV2 plays a direct pathogenetic role in $\mathrm{HF}{ }^{[15,16]}$. According to the inconsistent associations of COVID-19 with HF, a clear casual association between HF and COVID-19 infection is needed to further explore. 
Mendelian randomization (MR) has become an analytic method that probing genetic proxies effectively associated with exposures, and subsequently finding the association with outcomes through an intermediate trait. Moreover, MR plays an essential role in disentangling real causal effects from fallacious associations attributed by confounding and reverse causation bias ${ }^{[17,18]}$. If there is a causal relationship between the exposures and the outcomes, the genetic variation affecting the exposures will also affect the outcomes. MR has been considered to be a natural mimic of randomized controlled trials, so it has also been used in cardiovascular research, including heart failure, to detect new underlying etiological mechanisms and improve our understanding of current treatment methods ${ }^{[19,20]}$. This study aimed to identify common single nucleotide polymorphisms (SNPs) that affect COVID-19 infection in patients with COVID-19 and to employ the technique of MR to improve the understanding of the possible causality between COVID-19 infection and HF.

\section{Materials And Methods}

\section{SNP identification and data sources}

\section{Genetic association datasets for COVID-19}

These summarized data were derived from genome-wide association studies (GWAS) analysis of the latest version about the COVID-19 host genetics program from the UK Biobank individuals, which was aimed to determine the genetic determinants of COVID-19 susceptibility and the level of severity (https://www.covid19hg.org/results/) [21]. Including 3,523 patients and 36,634 control participants, 105 studies about COVID-19 have joined this program. These studies are carried out all over the world, including countries in Europe, America, Asia, and Africa.

\section{Genetic association datasets for heart failure}

We obtained the association between the specific SNPs and HF from the current largest GWAS metaanalysis of HF in people of European descent ${ }^{[22]}$. The GWAS meta-analysis, which was conducted by the Heart Failure Molecular Epidemiology for Therapeutic Targets Consortium included 26 studies (17 population cohorts studies, $38,780 \mathrm{HF}$ cases, 893,657 controls and 9 case-control studies, 8,529 cases, 36,357 controls), 47,309 patients with heart failure, and 930,014 control group [22]. This GWAS metaanalysis was adjusted according to gender, age, and main components. In all cohort studies, heart failure was assessed using at least one of the following methods: discharge registration, cause of death registration, or physician decision/diagnosis. Due to insufficient power, GWAS was not stratified according to etiological subtypes. All participants obtained informed consent, and the related ethics committees all approved these studies.

\section{MR analysis}

In the main analysis of MR analysis, we used the standard inverse-variance weighted (IVW) method to estimate the overall causal relationship between COVID-19 susceptibility to HF ${ }^{[23]}$. By using this method, 
the causal effect of exposure on the outcome is estimated as the ratio of the SNP outcomes associated with the exposures (Wald estimate). According to Mendel's law of inheritance, MR assumes that SNPs are randomly distributed in the general population (separation, independent classification), simulating the process of randomization, SNP always appears before the disease, so reverse causality can be effectively eliminated. To ensure a valid inverse weighted variance method within the MR analysis process, three significant assumptions need to be proved: (1) the SNPs have a robustly relation with COVID-19 (the exposure), (2) the SNPs are in complete independence from any potential confounding factors that influence both COVID-19 and HF, and (3) the SNPs exert influence on HF (the outcome) only by COVID-19 (the exposure) and not via any alternative causal pathways (Figure 1) [24, 25].

The standardized utilization of MR is a single-sample MR, conducted in a group of people, including intact data on the SNPs, exposure, and results of all participants ${ }^{[19]}$. According to the rare statistics in single-sample MR, the two-sample MR was developed to enable analysis in two independent samples, one for focusing on exposures and the other for outcomes ${ }^{[24]}$. In our study, we performed the IVW, the Mendelian randomization-Egger (MR-Egger) regression, the simple mode (SM), weighted median and weighted mode. These five two-sample MR methods were performed by the "Two-sample MR" package in $R$ (version 4.0.3) ${ }^{[23,26]}$. The related analysis was all one-sided, and the evidence of causal relationship was announced when a pre-specified p-value is lower than 0.05 .

According to the superiorities of each MR, these five means can make up a deficiency of each other and provide a more credible causal relationship for our research. In a two-sample MR analysis, we applied the IVW method to analyze the associations between genetically predicted COVID-19 infection and HF. The MR-Egger method was used for the estimation of accidental effects and evaluation of directed pleiotropy under weaker assumptions. When $50 \%$ or more of the genetic variations are valid instrumental variables, the median-based method can give a reliable effect estimate, which may be more suitable than the MREgger method. The weighting method provides a more accurate causal estimation based on more weight analysis. The simple mode help to avoid the effect of pleiotropic effects in causal reasoning and omit part of the genetic variation from the analysis. Compared with the traditional MR analysis, the robust method estimates the causal effects consistently under weaker assumptions.

Traditional IVW methods are the appropriate method to use aggregated data from GWAS. We used it to initially estimate the impact of COVID-19 on HF ${ }^{[23]}$. Firstly, we performed the IVW average of SNP-specific associations with fixed effects in HF. If some tools in the causal reasoning hypothesis based on MR analysis are invalid, the analysis gives a biased estimate ${ }^{[27]}$. Secondly, we resolved the first hypothesis (the true relation between SNPS and COVID-19) by choosing SNPS that robustly predicts COVID-19, and the gene variants that act as COVID-19 agents are likely to satisfy the second hypothesis (there are no confounding factors). We employed the MR-Egger regression to investigate directional pleiotropy to evaluate the potential violation of the third assumption. Subsequently, we created a scatter plot to visually detect the potential pleiotropy by showing the association between each SNP and HF risk against 
associated with COVID-19. Sensitivity analysis were conducted by using the MR-Egger regression, and weighted median, to explain the potential violation of the effective tool variable hypothesis.

\section{Results}

Causal association of COVID-19 with HF via forwarding MR. In the current MR analysis, we employed 29 independent SNPs of COVID-19 as the instrumental variables. The associations of genetically predicted COVID-19 with HF based on the IVW method are presented in Table 1. The odds ratio (OR) with a horizontal line shown in the forest plot (Fig. 2) represents the HF risk of COVID-19-related SNP alleles with $95 \%$ confidence intervals (Cls). The results of the present two-sample MR study showed that COVID-19 was associated with HF as risk factors ( $p=0.005$ for $r s 116736195$ and $p=0.005$ for rs 147516281 ), however, the total impact of 29 SNPs was negative. 
Table 1

Summarized data for the genetic variants associated with COVID-19.

\begin{tabular}{|c|c|c|c|c|c|c|}
\hline SNP & Chr. & 'Short' Allele & ‘Other' Allele & Beta Estimate & SE & Discovery $p$ \\
\hline rs3136704 & 1 & A & G & -0.0152 & 0.0125 & 0.2238 \\
\hline rs3136705 & 1 & $\mathrm{~T}$ & $\mathrm{C}$ & -0.0146 & 0.0125 & 0.2445 \\
\hline rs57136622 & 1 & $\mathrm{~T}$ & C & -0.0121 & 0.0125 & 0.334 \\
\hline rs2093932 & 1 & $\mathrm{~T}$ & C & -0.0057 & 0.0264 & 0.83 \\
\hline rs112340499 & 1 & C & G & -0.0131 & 0.0189 & 0.4889 \\
\hline rs73121428 & 1 & $\mathrm{~T}$ & $\mathrm{C}$ & 0.0036 & 0.0198 & 0.8545 \\
\hline rs12133284 & 1 & $\mathrm{~T}$ & $A$ & -0.0057 & 0.0105 & 0.5868 \\
\hline rs6736835 & 2 & A & C & 0.0039 & 0.0194 & 0.8388 \\
\hline rs116513329 & 2 & $\mathrm{~T}$ & C & 0.0081 & 0.0257 & 0.7517 \\
\hline rs56248709 & 3 & $\mathrm{~T}$ & $\mathrm{C}$ & 0.0058 & 0.0119 & 0.625 \\
\hline rs12641246 & 4 & A & C & -0.0022 & 0.0129 & 0.8653 \\
\hline rs184148704 & 4 & A & G & -0.0195 & 0.047 & 0.6784 \\
\hline rs181349760 & 4 & A & G & -0.0242 & 0.0354 & 0.4941 \\
\hline rs147516281 & 5 & $\mathrm{~T}$ & $\mathrm{C}$ & 0.0708 & 0.0253 & 0.005079 \\
\hline rs116736195 & 5 & A & G & 0.0708 & 0.0253 & 0.005079 \\
\hline rs150253438 & 6 & $\mathrm{~T}$ & G & -0.0122 & 0.032 & 0.7024 \\
\hline rs77101489 & 6 & A & G & 0.0224 & 0.0355 & 0.5277 \\
\hline rs147259357 & 6 & A & $\mathrm{T}$ & -0.0218 & 0.0513 & 0.6707 \\
\hline rs62436931 & 6 & C & $\mathrm{T}$ & -0.0028 & 0.0149 & 0.8501 \\
\hline rs17092129 & 8 & A & G & -0.022 & 0.0401 & 0.5837 \\
\hline rs143530761 & 10 & $\mathrm{~T}$ & C & 0.0282 & 0.0274 & 0.3032 \\
\hline rs80066318 & 13 & $\mathrm{~T}$ & C & 0.0001 & 0.0339 & 0.998 \\
\hline rs112981919 & 15 & $\mathrm{~T}$ & C & -0.017 & 0.0627 & 0.7869 \\
\hline rs75631133 & 16 & C & $\mathrm{T}$ & 0.0006 & 0.0392 & 0.9877 \\
\hline rs35746115 & 17 & A & C & 0.0074 & 0.0095 & 0.4366 \\
\hline rs12950851 & 17 & $\mathrm{~T}$ & C & 0.009 & 0.0097 & 0.352 \\
\hline
\end{tabular}




\begin{tabular}{|lllllll|}
\hline SNP & Chr. & 'Short' Allele & 'Other' Allele & Beta Estimate & SE & Discovery $\boldsymbol{p}$ \\
\hline rs13346571 & 19 & G & A & 0.0059 & 0.0081 & 0.4611 \\
\hline rs8116527 & 20 & A & G & -0.0048 & 0.0089 & 0.5923 \\
\hline rs118178636 & 22 & T & G & -0.0144 & 0.0299 & 0.6294 \\
\hline SNP: single nucleotide polymorphism; Chr.: chromosome; SE: standard error. & \\
\hline
\end{tabular}

The scatter plot (Fig. 3) shows the association of each allele with heart failure and corresponds to one standard deviation of COVID-19 (the $95 \% \mathrm{Cl}$ of each SNP is represented by vertical and horizontal black lines). Odds ratios for the association between COVID-19 and HF using different methodological approaches are presented in Table 2.

Table 2

The association between COVID-19 and HF of odds ratios using different methodological approaches.

\begin{tabular}{|llll|}
\hline & OR $(95 \%$ Cl) & P & SE \\
\hline IVW & $1.004(0.994,1.014)$ & 0.467 & 0.005 \\
\hline MR-Egger & $1.008(0.996,1.019)$ & 0.218 & 0.006 \\
\hline SM & $0.968(0.924,1.015)$ & 0.186 & 0.024 \\
\hline Weighted Median & $1.001(0.988,1.014)$ & 0.881 & 0.007 \\
\hline Weighted Mode & $1.001(0.989,1.014)$ & 0.836 & 0.007 \\
\hline $\begin{array}{l}\text { IVW: the inverse variance weighted method; MR-Egger: the Mendelian randomization-Egger method; } \\
\text { SM: simple mode; OR: odds ratio; Cl: confidence interval; P: p-value; SE: standard error. }\end{array}$ \\
\hline
\end{tabular}

IVW assessment demonstrated there was no relation between the genetically instrumented COVID-19 risk and HF (OR, 1.004; $95 \% \mathrm{Cl}, 0.994-1.014 ; P=0.467)$, without obvious heterogeneity $(P=0.599)$. There were also no associations when using the weighted median method (ORs, $1.001 ; 95 \% \mathrm{Cl}, 0.988-1.014)$. In the analysis, we used estimates based on weighted modes, and the results showed that the ORs were 1.001 (95\% Cl 0.989-1.014). The MR-Egger regression analysis did not reveal any signs of directional pleiotropy for COVID-19 (intercept=-0.004; $p=0.673$ ), and provided no evidence to support the causality between COVID-19 and HF (OR, 1.008; $95 \% \mathrm{Cl} 0.996$ to 1.019; $\mathrm{P}=0.218)$. An analysis using the simple mode-based estimate, the ORs were 0.967 (95\% $\mathrm{Cl} 0.924-1.015)$. In general, the above five MR detection models all showed that there is no causal relationship between COVID-19 and HF.

\section{Discussion}


To make known the causality of COVID-19 on HF, we performed Mendelian randomization analysis of these two samples using publicly aggregated GWAS data. With genetic variants as proxies for COVID-19, the present study only showed COVID-19 was correlated with HF to a certain extent; however, no causal effect of COVID-19 on HF risk was observed. Although the association between COVID-19 and HF is unlikely to be causal, infection of COVID-19 might be a predictor for the incidence of HF in the general population.

Some retrospective studies have shown that after acute respiratory distress syndrome (ARDS), respiratory failure, and sepsis, HF was the most frequent clinical manifestation of COVID-19 ${ }^{[9,28]}$. As a possible severe consequence of COVID-19 related myocardial injury ${ }^{[29,30]}$, HF is accompanied by high mortality [13]. Meanwhile, HF patients are at especially increased risk due to their reduced immunity, general frailty, and reduced hemodynamic ability to cope with more severe infections. It was reported that in HF patients, monocytes seem to produce more tumor necrosis factor a (TNF- $a$ ) and less interleukin-10(IL-10) than healthy subjects ${ }^{[31]}$. TNF-a and IL-10 play different roles in the process of inflammation. TNF-a is one of the most important pro-inflammatory cytokines. It regulates blood coagulation, promotes oxidative stress in inflammatory areas, and indirectly causes fever ${ }^{[32]}$. The IL-10, a cytokine with anti-inflammatory properties, plays a central role in infection by limiting the immune response to pathogens and preventing damage to the host ${ }^{[33]}$. Patients with cardiovascular diseases are more prone generally to a viral illness and thus constitute already a high-risk group. Despite previous observational studies showing an association between COVID-19 and HF, such relevance has not been firmly endorsed whether COVID-19 plays a causal role in HF. MR study, an IV-based method to infer the causality between intermediate phenotypes and disease, has been widely conducted in HF research ${ }^{[22]}$.

Although a few cases of direct virus-related myocarditis and may subsequently result in HF, other mechanisms are responsible for myocardial injury or HF in the most of COVID-19 patients, rather than direct viral infection alone ${ }^{[15,29,34-36]}$. So far, few cases of COVID-19 related acute myocarditis are described in the literature. In some cases, SARS-CoV-2 was shown within macrophages, but not in cardiomyocytes. Furthermore, endomyocardial biopsy showed only low-grade interstitial myocardial inflammation and specific changes of cardiac myocytes with myofibrillar lysis and lipid droplets ${ }^{\text {[37] }}$. These findings showed the virus could reside within the heart but does not prove that it has a direct pathogenetic role in $\mathrm{HF}^{[15,16]}{ }^{[}$. Our novel results firstly indicate that COVID-19 has no causal effects on the risk of HF, which are consistent with previous studies ${ }^{[15,16]}$. These findings implied the physicians should treat COVID-19 as genuine confounders and pay more attention to other factors in high-risk individuals.

Our MR research has several advantages. First, this is the first study of the causal relationship between COVID-19 and HF. Our study investigated the largest GWAS meta-analysis of heart failure with a total of 47,309 cases and 930,014 controls, which minimizes the impact of population stratification. Second, we performed five complementary MR methods to prevent reverse causal bias and to limit potential confounding. The result of this study was a robust multiple sensitivity analysis showing that 
confounding is unlikely to explain the observed association. Third, multiple SNPs are used as a tool to study COVID-19, which makes it possible to detect HF risk and provide a powerful genetic tool for COVID19 infection.

Some potential limitations also exist in our study. First, we cannot effectively evaluate the compensation mechanism, which weakens the estimate and thus reduces the accuracy. Second, the GWAS metaanalysis of HF is limited to European descent, thus hindering universality in the entire population. Third, although we applied several methods to try to rule out pleiotropy, the association between SNPs and COVID-19 may still be through other means. Nor can we rule out the common genetic basis between COVID-19 and HF. These results are only derived from statistical analysis, so doctors must be more cautious about patients with COVID-19. As a factor for cardiac injury, COVID-19 has proven its value and

even represents a predictor for $\mathrm{HF}$ risk ${ }^{[5,9]}$. Thus, although our analysis provided no evidence for a causal association between COVID-19 and HF, it did not preclude the value of COVID-19 infection in HF risk prediction. In the future, more research on COVID-19 and heart failure is needed to explore the correlation between COVID-19 and HF from different perspectives.

\section{Conclusions}

Our MR analysis uses COVID-19 related SNPs as an instrumental variable extracted from the GWAS data. The results of the current MR analysis do not support the causal effect of COVID-19 infection (exposures) on HF (outcome variables). Further bidirectional MR research needs to use data based on individual levels of different races to elaborate the potential causal relationship between COVID-19 infection and the risk of HF.

\section{Abbreviations}

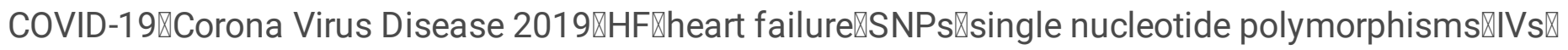

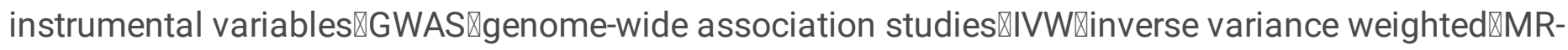

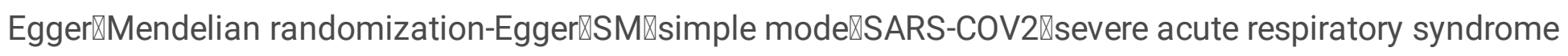

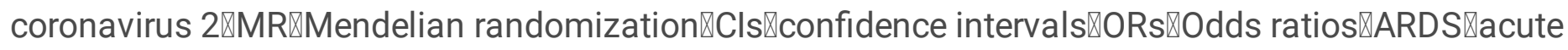
respiratory distress syndrome囚TNF-a冈tumor necrosis factor $\mathrm{a} \bigotimes \mathrm{IL}-10 \bigotimes$

\section{Declarations}

\section{Acknowledgements}

The authors are grateful for the constructive comments from anonymous reviewers of this publication. We thank all the authors and participants of all GWASs, from which we used aggregate statistics. The authors are solely responsible for the design and implementation of this research, all research analysis, the drafting and editing of the paper, and its final contents.

\section{Authors' Contributors}


HW and ZG conceived and designed the study. HW, ZG and $Y Z$ searched the literature, collected and analyzed the data. HW, ZG and BC contributed to the writing of the manuscript.

\section{Funding}

None.

\section{Availability of data and materials}

The data used to support the findings of this study are included within the article.

\section{Ethics approval and consent to participate}

Not applicable.

\section{Consent for publication}

Not applicable.

\section{Competing interests}

The authors declare that there are no competing interests.

\section{References}

1. Dong E, Du H, Gardner L. An interactive web-based dashboard to track COVID-19 in real time. Lancet Infect Dis 2020; 20(5):533-534.

2. Huang C, Wang Y, Li X, Ren L, Zhao J, Hu Y, et al. Clinical features of patients infected with 2019 novel coronavirus in Wuhan, China. Lancet 2020; 395(10223):497-506.

3. Chen N, Zhou M, Dong X, Qu J, Gong F, Han Y, et al. Epidemiological and clinical characteristics of 99 cases of 2019 novel coronavirus pneumonia in Wuhan, China: a descriptive study. Lancet 2020; 395(10223):507-513.

4. Böhm M, Frey N, Giannitsis E, Sliwa K, Zeiher AM. Coronavirus Disease 2019 (COVID-19) and its implications for cardiovascular care: expert document from the German Cardiac Society and the World Heart Federation. Clin Res Cardiol 2020.

5. Shi S, Qin M, Shen B, Cai Y, Liu T, Yang F, et al. Association of Cardiac Injury With Mortality in Hospitalized Patients With COVID-19 in Wuhan, China. JAMA Cardiol 2020; 5(7):802-810.

6. Arentz M, Yim E, Klaff L, Lokhandwala S, Riedo FX, Chong M, et al. Characteristics and Outcomes of 21 Critically III Patients With COVID-19 in Washington State. JAMA 2020; 323(16):1612-1614.

7. Guo T, Fan Y, Chen M, Wu X, Zhang L, He T, et al. Cardiovascular Implications of Fatal Outcomes of Patients With Coronavirus Disease 2019 (COVID-19). JAMA cardiology 2020; 5(7):811-818. 
8. Rey JR, Caro-Codón J, Rosillo So, Iniesta ÁM, Castrejón-Castrejón S, Marco-Clement I, et al. Heart Failure In Covid-19 Patients: Prevalence, Incidence And Prognostic Implications. European journal of heart failure 2020.

9. Zhou F, Yu T, Du R, Fan G, Liu Y, Liu Z, et al. Clinical course and risk factors for mortality of adult inpatients with COVID-19 in Wuhan, China: a retrospective cohort study. Lancet 2020; 395(10229):1054-1062.

10. Chen L, Li X, Chen M, Feng Y, Xiong C. The ACE2 expression in human heart indicates new potential mechanism of heart injury among patients infected with SARS-CoV-2. Cardiovasc Res 2020; 116(6):1097-1100.

11. Zhang P, Zhu L, Cai J, Lei F, Qin J-J, Xie J, et al. Association of Inpatient Use of AngiotensinConverting Enzyme Inhibitors and Angiotensin II Receptor Blockers With Mortality Among Patients With Hypertension Hospitalized With COVID-19. Circ Res 2020; 126(12):1671-1681.

12. Mehta P, McAuley DF, Brown M, Sanchez E, Tattersall RS, Manson JJ. COVID-19: consider cytokine storm syndromes and immunosuppression. Lancet 2020; 395(10229):1033-1034.

13. Liu PP, Blet A, Smyth D, Li H. The Science Underlying COVID-19: Implications for the Cardiovascular System. Circulation 2020; 142(1):68-78.

14. Sama IE, Ravera A, Santema BT, van Goor H, Ter Maaten JM, Cleland JGF, et al. Circulating plasma concentrations of angiotensin-converting enzyme 2 in men and women with heart failure and effects of renin-angiotensin-aldosterone inhibitors. Eur Heart J 2020; 41(19):1810-1817.

15. Clerkin KJ, Fried JA, Raikhelkar J, Sayer G, Griffin JM, Masoumi A, et al. COVID-19 and Cardiovascular Disease. Circulation 2020; 141(20):1648-1655.

16. Zhou R. Does SARS-CoV-2 cause viral myocarditis in COVID-19 patients? Eur Heart J 2020; 41(22):2123.

17. Smith GD, Ebrahim S. 'Mendelian randomization': can genetic epidemiology contribute to understanding environmental determinants of disease? Int J Epidemio/ 2003; 32(1).

18. Lawlor DA, Harbord RM, Sterne JAC, Timpson N, Davey Smith G. Mendelian randomization: using genes as instruments for making causal inferences in epidemiology. Stat Med 2008; 27(8):11331163.

19. Wensley F, Gao P, Burgess S, Kaptoge S, Di Angelantonio E, Shah T, et al. Association between C reactive protein and coronary heart disease: mendelian randomisation analysis based on individual participant data. BMJ 2011; 342:d548.

20. Swerdlow DI, Preiss D, Kuchenbaecker KB, Holmes MV, Engmann JEL, Shah T, et al. HMG-coenzyme A reductase inhibition, type 2 diabetes, and bodyweight: evidence from genetic analysis and randomised trials. Lancet 2015; 385(9965):351-361.

21. The COVID-19 Host Genetics Initiative, a global initiative to elucidate the role of host genetic factors in susceptibility and severity of the SARS-CoV-2 virus pandemic. Eur J Hum Genet 2020; 28(6):715718. 
22. Shah S, Henry A, Roselli C, Lin H, Sveinbjörnsson G, Fatemifar G, et al. Genome-wide association and Mendelian randomisation analysis provide insights into the pathogenesis of heart failure. Nat Commun 2020; 11(1):163.

23. Burgess S, Butterworth A, Thompson SG. Mendelian randomization analysis with multiple genetic variants using summarized data. Genet Epidemio/ 2013; 37(7):658-665.

24. Burgess S, Scott RA, Timpson NJ, Davey Smith G, Thompson SG. Using published data in Mendelian randomization: a blueprint for efficient identification of causal risk factors. Eur J Epidemiol 2015; 30(7):543-552.

25. Grover S, Del Greco M F, Stein CM, Ziegler A. Mendelian Randomization. Methods Mol Biol 2017; 1666:581-628.

26. Yavorska 0O, Burgess S. MendelianRandomization: an R package for performing Mendelian randomization analyses using summarized data. Int J Epidemiol 2017; 46(6):1734-1739.

27. Didelez V, Sheehan N. Mendelian randomization as an instrumental variable approach to causal inference. Stat Methods Med Res 2007; 16(4):309-330.

28. Chen T, Wu D, Chen H, Yan W, Yang D, Chen G, et al. Clinical characteristics of 113 deceased patients with coronavirus disease 2019: retrospective study. BMJ 2020; 368:m1091.

29. Madjid M, Safavi-Naeini P, Solomon SD, Vardeny O. Potential Effects of Coronaviruses on the Cardiovascular System: A Review. JAMA cardiology 2020; 5(7):831-840.

30. Babapoor-Farrokhran S, Gill D, Walker J, Rasekhi RT, Bozorgnia B, Amanullah A. Myocardial injury and COVID-19: Possible mechanisms. Life Sci 2020; 253:117723.

31. $\mathrm{Ng} \mathrm{TM}$, Toews ML. Impaired norepinephrine regulation of monocyte inflammatory cytokine balance in heart failure. World journal of cardiology 2016; 8(10):584-589.

32. Zelová H, Hošek J. TNF-a signalling and inflammation: interactions between old acquaintances. Inflammation research : official journal of the European Histamine Research Society [et al] 2013; 62(7):641-651.

33. Saraiva M, O'Garra A. The regulation of IL-10 production by immune cells. Nature reviews Immunology 2010; 10(3):170-181.

34. Kochi AN, Tagliari AP, Forleo GB, Fassini GM, Tondo C. Cardiac and arrhythmic complications in patients with COVID-19. J Cardiovasc Electrophysio/ 2020; 31(5):1003-1008.

35. Peretto G, Sala S, Caforio ALP. Acute myocardial injury, MINOCA, or myocarditis? Improving characterization of coronavirus-associated myocardial involvement. Eur Heart J 2020; 41(22):21242125.

36. Guzik TJ, Mohiddin SA, Dimarco A, Patel V, Savvatis K, Marelli-Berg FM, et al. COVID-19 and the cardiovascular system: implications for risk assessment, diagnosis, and treatment options. Cardiovasc Res 2020; 116(10):1666-1687.

37. Sala S, Peretto G, Gramegna M, Palmisano A, Villatore A, Vignale D, et al. Acute myocarditis presenting as a reverse Tako-Tsubo syndrome in a patient with SARS-CoV-2 respiratory infection. Eur 
Heart J 2020; 41(19):1861-1862.

Figures

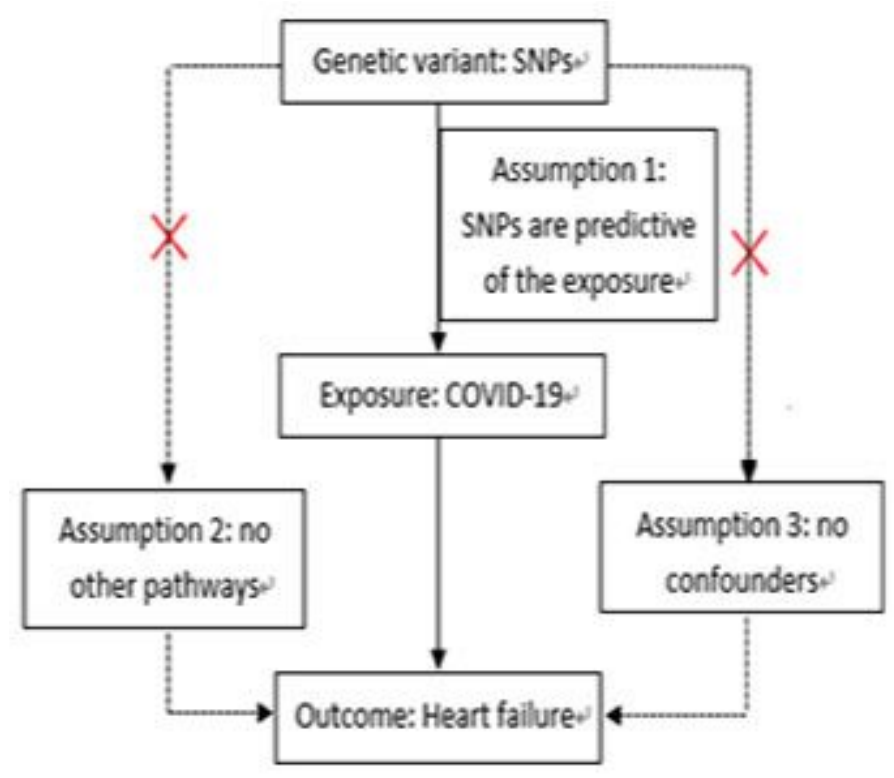

Figure 1

MR analysis

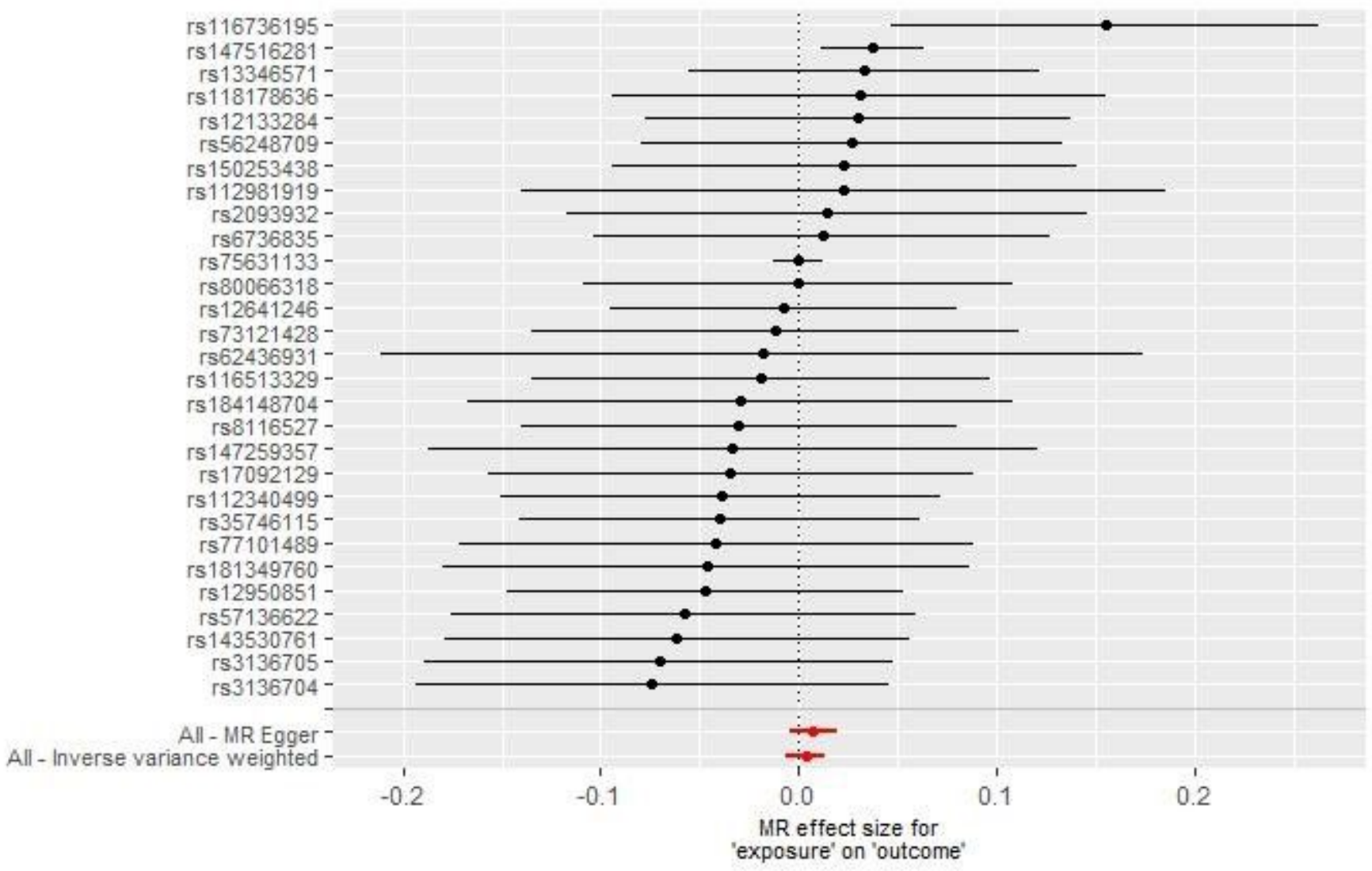

Figure 2 
The odds ratio (OR) with a horizontal line shown in the forest plot represents the HF risk of COVID-19related SNP alleles with $95 \%$ confidence intervals (Cls).

MR Test
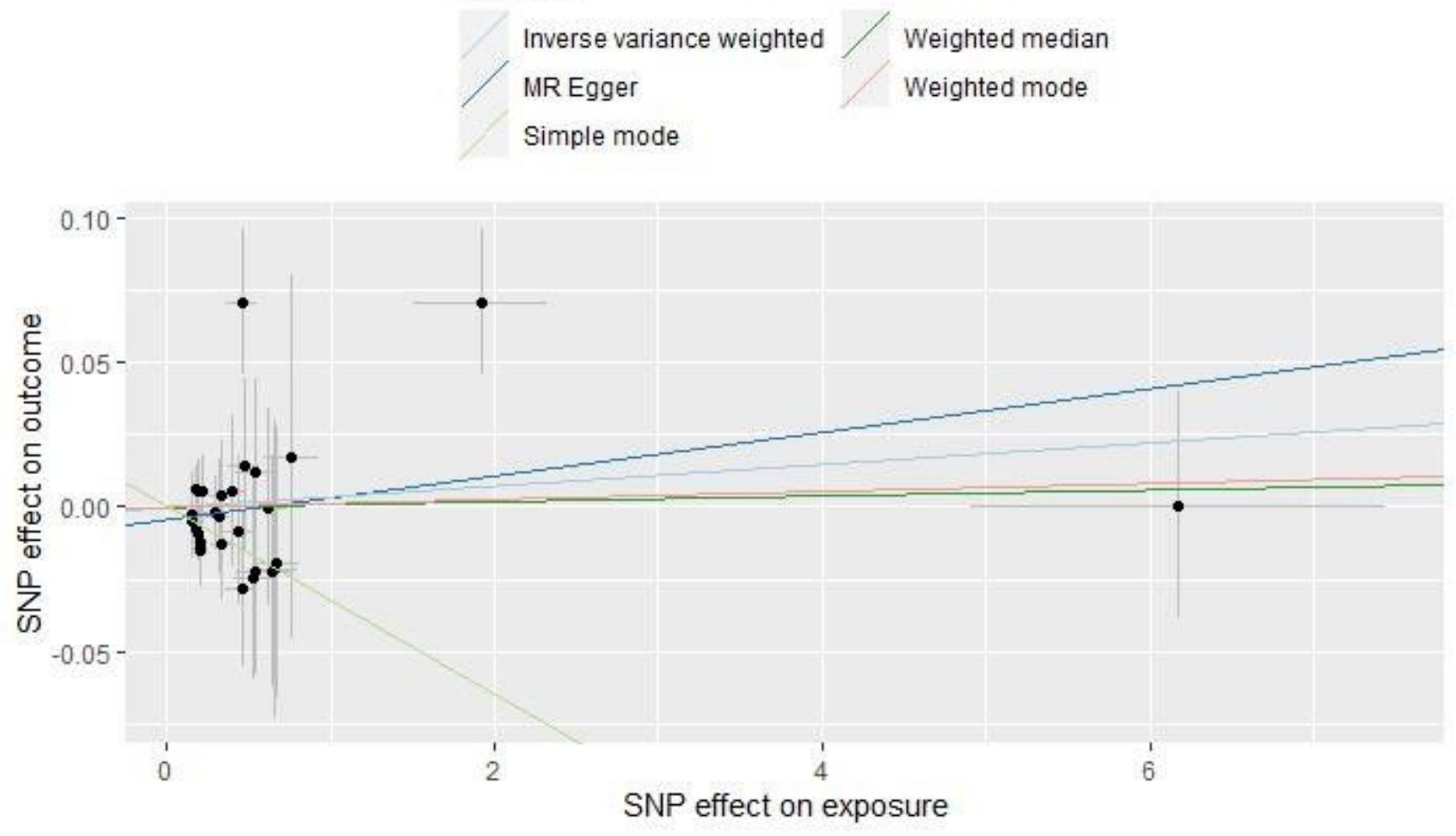

\section{Figure 3}

The scatter plot shows the association of each allele with heart failure and corresponds to one standard deviation of COVID-19 